\title{
QUALITY-OF-SERVICE SPECIFIC INFORMATION RETRIEVAL FOR DENSELY DEPLOYED SENSOR NETWORKS
}

\author{
Qing Zhao and Lang Tong \\ School of Electrical and Computer Engineering \\ Cornell University, Ithaca, NY 14853 \\ \{qzhao,ltong\}@ece.cornell.edu
}

\begin{abstract}
A new MAC protocol is proposed for the reachback operation in large scale, densely deployed sensor networks. Referred to as QUality-of-service specific Information REtrieval (QUIRE), the proposed protocol aims to assure QoS requirement with a minimum amount of transmissions from sensors. By enabling only one sensor in a neighborhood to transmit, QUIRE ensures that the data access point receives no redundant information for reconstructing the sensed field within a given maximum distortion $(Q o S)$. It jointly minimizes system latency (the amount of time spent for data collection) and total energy consumption (the total number of transmissions from sensors).
\end{abstract}

\section{INTRODUCTION}

\subsection{Sensor Networks with Mobile Agents}

A new network architecture, referred to as SEnsor Networks with Mobile Agents (SENMA), is proposed in [1] for low power, large scale sensor networks. As illustrated in Figure 1, SENMA consists of two types of nodes: sensors and mobile agents. Sensors are low power and low cost nodes that are limited in processing and communication capability. They are deployed in large quantities, and often through random aerial drop. On the contrary, mobile agents are equipped with powerful processors and sophisticated transceivers. They have unlimited power supply and the capability of traversing the sensor network with carefully designed trajectory. Examples of mobile agents include manned or unmanned aerial vehicles, ground vehicles equipped with sophisticated terminals and power generators, or specially designed light nodes that can hop around in the network. These mobile agents may have high data rate connection to satellites, allowing reachback to remote command control centers.

In SENMA, sensors communicate directly with the mobile agents. This avoids much of the overhead associated with medium access control and routing. Compared with the ad hoc architecture where sensors themselves are responsible for organizing medium access control, discovering and maintaining routes, storing and relaying packets, encoding and decoding, SENMA, by shifting most of the processing burden from sensor nodes to a few

\footnotetext{
${ }^{0}$ This work was supported in part by the Multidisciplinary University Research Initiative (MURI) under the Office of Naval Research Contract N00014-00-10564, and Army Research Laboratory CTA on Communication and Networks under Grant DAAD19-01-2-0011.
}

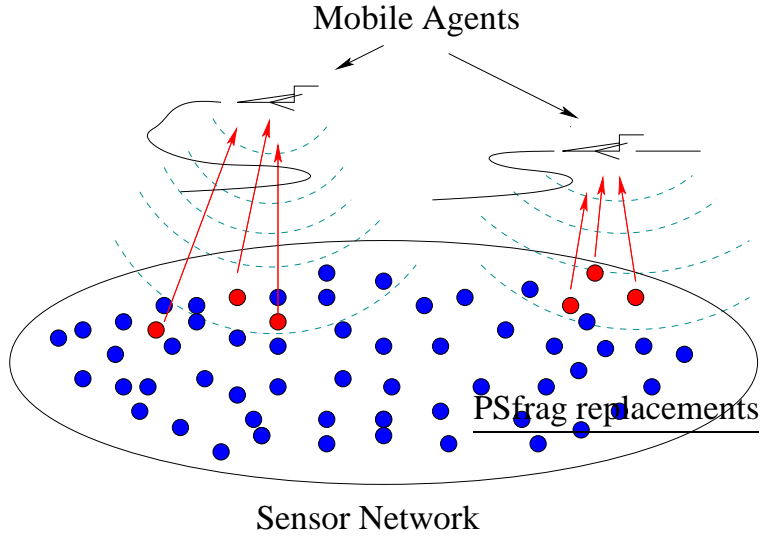

Fig. 1: Sensor Networks with Mobile Agents

powerful mobile agents, provides an attractive alternative that is simple to maintain and easy to scale.

\subsection{Medium Access Control in SENMA}

Consider a sensor network deployed to monitor the radiation level around a nuclear laboratory in a remote area. Each sensor is preprogrammed to sense the radiation level at specific time instants. During the reachback operation, an airplane flies to the sensor field to collect data. Sensors then transmit their data to this mobile agent through a common wireless channel. The problem we have here is, as one immediately recognizes, medium access control (MAC).

At the first glance, this problem seems well-studied - numerous MAC protocols have been proposed for communication networks where a number of users transmit to a central controller through a common medium. Two unique features of sensor networks, however, distinguish MAC design in SENMA from that for communication networks. Directly applying MAC protocols developed for communication networks to sensor networks can result in waste of network resource.

First, sensors are redundantly deployed to ensure converge and fault-tolerant performance; individual sensors may be placed in dead spots, in sleep, or nonfunctional due to power shortage. It is unnecessary, nor is it possible, for the mobile agent to receive every node's packet. Second, in the reachback operation where the mobile agent extracts information from the sensor network, the mobile agent knows that every node, when functioning, has 
a packet to transmit. This contracts sharply with conventional communication networks with random and busty packet arrivals.

The first feature clearly excludes the fixed allocation schemes where every node in the network is allocated with a share of network resource (in terms of, for example, time slot, frequency bin, or orthogonal spreading code) for its exclusive use. This leaves us with random access MAC protocols where nodes contend for channel access. Mainly designed to handle random and busty traffic, random access protocols are incompatible with the second feature of the reachback operation in sensor networks. If we allow all sensors, each holding a packet that can be strongly correlated with other sensors' data, to contend for channel access, many redundant transmissions occur, causing excessive interference and unnecessary energy expenditure.

\subsection{QoS Specific Information Retrieval}

Then how should we design MAC protocols for the reachback operation in densely deployed sensor networks? First, we should eliminate unnecessary transmissions which provides nothing but interference. Second, MAC protocols designed for sensor networks should be energy-aware to prolong network lifetime.

In this paper, we propose a MAC protocol for the reachback operation in sensor networks. Referred to as QUality-of-service specific Information REtrieval (QUIRE), the proposed protocol assures quality-of-service (QoS) requirement with a minimum amount of transmissions from sensors. Based on the density of deployment, auto-correlation of the sensed random field, and the QoS specification given as the maximum distortion for reconstructing the random field, QUIRE partitions the sensor network into disjoint and equal-sized cells. It completely eliminates redundant transmissions by enabling only one sensor in each cell to transmit. It provides energy-awareness by giving higher priority to sensors with high energy supply. Built upon a multi-user physical layer which allows simultaneous packet receptions at the mobile agent, QUIRE jointly minimizes system latency (the amount of time the mobile agent stays in the sensor field) and total energy consumption (the total number of transmissions from sensors).

\subsection{Related Work}

Besides SENMA, two architectures have been considered for sensor networks, namely, flat ad hoc and hierarchical ad hoc. The main theme of research activities on MAC protocol design for sensor networks is based on the flat ad hoc architecture [2-5]. In [6], a hierarchical ad hoc architecture where sensors form clusters and only the cluster heads are responsible for relaying packets to a fixed remote base station is proposed. MAC protocols are developed [7] for the transmission from sensors to cluster heads and a follow-up work can be found in [8]. Perhaps [9] and [10] are the most relevant work to this paper. In [9], the authors consider direct transmission from sensors to a fixed remote base station and explicitly exploit node redundancy. They develop an adaptive scheme for each sensor to determine independently whether to transmit or not so that a fixed total number of transmissions occur in each slot. The difference between [9] and our work is that QoS in [9] is defined as the total number of transmissions that should occur in each slot and an independent channel from each sensor to the remote base station seems to be assumed by the authors. In [10], an ALOHA-based random access protocol is proposed for the reachback operation in SENMA. The novelty of [10] lies in the use of channel state information characterized by the propagation channel gain. It is shown in [10] that an asymptotic throughput no smaller than the spreading gain of the system can be achieved with arbitrarily small power in each sensor if the channel state information is used for determining each sensor's transmission probability. Different from QUIRE, the protocol proposed in [10] does not address QoS issues. Being a sensor-initiated random access protocol, it does not eliminate redundant transmissions.

\section{PROBLEM STATEMENT}

Consider the example we give earlier where a sensor network is deployed to monitor the radiation level in a remote area which forms a random field. Without loss of generality, we assume this remote area is a unit-area disk centered at the origin with $\mathcal{D}$ denoting the set of points on this disk, and $S(\mathcal{D})$ the random field. We pose the following assumptions on $S(\mathcal{D})$.

A1 $S(x, y)$ for all $(x, y) \in \mathcal{D}$ have common mean and variance.

$$
E[S(x, y)]=\mu, \quad E\left[(S(x, y)-\mu)^{2}\right]=\sigma^{2} .
$$

A2 The correlation function of $S(\mathcal{D})$ is spatially homogeneous.

$$
\begin{aligned}
& R((x, y),(u, v)) \triangleq E[(S(x, y)-\mu)(S(u, v)-\mu)] \\
& =R(d((x, y),(u, v))),
\end{aligned}
$$

where $d((x, y),(u, v)))$ is the Euclidean distance between $(x, y)$ and $(u, v)$.

A3 $R(d)$ is continuous and monotonically decreasing on [0,2].

The distribution of sensors on $\mathcal{D}$ forms a two-dimensional Poisson field with mean $\lambda$ nodes $/ \mathrm{m}^{2}$, i.e., the number $N(A)$ of sensors within an area of $A \mathrm{~m}^{2}$ is a Poisson random variable with mean $\lambda A$. By the total randomness of Poisson distribution, given $N(A)=k$, these $k$ sensors are uniformly distributed within this area of $A \mathrm{~m}^{2}$.

If a sensor locates at $(x, y)$, it measures the value (one realization) of $S(x, y)$ and generates a packet containing its measurements to be transmitted to the mobile agent. Note that the packet generated by a particular sensor should also contain the location of that sensor to make its measurements usable. For this type of application, sensors must acquire their locations via GPS or other position estimation schemes [11-13] after deployment.

In the reachback operation, the mobile agent flies to the sensor field to collect data. Sensors start to transmit their packets according to a specific MAC protocol. We assume that the transmission time is slotted based on the periodical beacon signals transmitted by the mobile agents. It will become clear later that the proposed MAC protocol requires only coarse slot synchronization among transmissions. The slotted channel from the sensor field to the mobile agent is characterized by $C_{n, k}$, the probability of having 
$k$ successes in a slot with $n$ transmissions. The multipacket reception (MPR) matrix of the channel is given by

$$
\mathbf{C}=\left(\begin{array}{ccccc}
C_{1,0} & C_{1,1} & & & \\
C_{2,0} & C_{2,1} & C_{2,2} & & \\
C_{3,0} & C_{3,1} & C_{3,2} & C_{3,3} & \\
\vdots & \vdots & \vdots & \vdots & \vdots
\end{array}\right)
$$

Let $\mathcal{C}_{n} \triangleq \sum_{k=1}^{n} k C_{n, k}$ be the expected number of correctly received packets when total $n$ packets are transmitted. We then define the capacity of the MPR channel as

$$
\eta \triangleq \max _{n=1, \cdots, \infty} \mathcal{C}_{n}
$$

Let

$$
n_{0} \triangleq \arg \max _{n=1, \cdots, M} \mathcal{C}_{n}
$$

We can see that $n_{0}$ packets should be transmitted simultaneously to achieve the channel capacity $\eta$.

We assume that the mobile agent can distinguish between empty and nonempty slots. If at least one packet is successfully demodulated at the end of a slot, the mobile agent does not assume the knowledge whether there are other packets transmitted in this slot but not successfully received.

After data collection, the mobile agent makes the data available to a remote control center where $S(\mathcal{D})$ is to be reconstructed. Let $\mathcal{A}$ denote all the points whose data are available to the control center. Let $\overline{\mathcal{A}}$ denote the complement of $\mathcal{A}$ in $\mathcal{D}$. Then the random field is reconstructed by approximate a point $\left(x_{0}, y_{0}\right)$ in $\overline{\mathcal{A}}$ with a point in $\mathcal{A}$ that is closest to $\left(x_{0}, y_{0}\right)$, i.e.,

$$
\hat{S}\left(x_{0}, y_{0}\right)=S\left(x_{1}, y_{1}\right)
$$

where

$$
\left(x_{1}, y_{1}\right)=\arg \min _{(x, y) \in \mathcal{A}} d\left(\left(x_{0}, y_{0}\right),(x, y)\right) .
$$

The QoS requirement is characterized by the maximum distortion $D$ in terms of mean square error (MSE) and the probability $P_{s}$ of successful reconstruction of $S(\mathcal{D})$, i.e., with a probability no smaller than $P_{s}$, every point in $\mathcal{D}$ can be estimated with an MSE no larger than $D$

$$
E\left[(\hat{S}(x, y)-S(x, y))^{2}\right] \leq D \quad \forall(x, y) \in \mathcal{D} .
$$

Here we assume errors caused by sensor's limited accuracy and quantization are negligible compared to the approximation error given in (4).

Our problem here is to design a MAC protocol that jointly minimizes system latency $L$ (the amount of time spent on data collection) and total number $U$ of transmissions for a given QoS requirement $\left(D, P_{s}\right)$.

\section{QOS SPECIFIC INFORMATION RETRIEVAL}

QUIRE consists of two steps. First, based on the density $\lambda$ of deployment, autocorrelation $R(d)$ of the random field, and the QoS requirement $\left(D, P_{s}\right)$, the mobile agent partitions $\mathcal{D}$ into disjoint and equal-sized cells. In the second step, one sensor from each cell is enabled according to a transmission control scheme so that the system latency $L$ and the total number $U$ of transmissions are jointly minimized.

\subsection{Partitioning $\mathcal{D}$ into cells}

Consider a point $\left(x_{0}, y_{0}\right)$ in $\mathcal{D}$. Let $\left(x_{1}, y_{1}\right)$ be the point in $\mathcal{A}$ that is closet to $\left(x_{0}, y_{0}\right)$. From (4) and (5), we have

$E\left[\left(\hat{S}\left(x_{0}, y_{0}\right)-S\left(x_{0}, y_{0}\right)\right)^{2}\right]=2 \sigma^{2}-2 R\left(d\left(\left(x_{0}, y_{0}\right),\left(x_{1}, y_{1}\right)\right)\right)$

To ensure a maximum MSE of $D$, we need

$$
R\left(d\left(\left(x_{0}, y_{0}\right),\left(x_{1}, y_{1}\right)\right)\right) \geq\left(2 \sigma^{2}-D\right) / 2 .
$$

Define

$$
r \triangleq \max \left(\left\{d: R(d) \geq\left(2 \sigma^{2}-D\right) / 2, d \in[0,2]\right\}\right) .
$$

It then follows that to estimate $S\left(x_{0}, y_{0}\right)$ with a maximum MSE of $D$, at least one sensor should be located at most $r$ away from $\left(x_{0}, y_{0}\right)$ and its packet should be received by the mobile agent. In another word, if the mobile agent receives a packet from the sensor located at $(x, y)$, it can reconstruct every point in the $r$ radius disk centered at $(x, y)$ with an MSE no larger than $D$. It follows that if we partition $\mathcal{D}$ into $r$-radius disks and collect one packet from the center of each disks, the whole field $S(\mathcal{D})$ can be estimated with a maximum distortion of $D$. Since we can not cover an area with disjoint disks without leaving gaps, we need to consider other geometric shapes to minimize the total number of cells, hence the total number of transmissions. The best choice, as we know from cellular systems, is to cover $\mathcal{D}$ with disjoint equal-sized hexagons.

Now the question is how large can each cell be. We know each cell should be contained by a $r$-radius disk to ensure a maximum distortion $D$. But can the radius of each hexagon be as large as $r$ ? In general, no. To have a cell size of $r$, we require that a sensor is located exactly at the center of each cell, which, unfortunately, is a zero-probability event for finite $\lambda$. To satisfy the QoS requirement $\left(D, P_{s}\right)$, the cell size should be smaller than $r$. Suppose we choose $\left(r-r_{0}\right)$ as the radius of each hexagon. Then all points inside a particular cell can be estimated with a maximum distortion $D$ if there locates a sensor inside the $r_{0}$-radius disk at the center of that cell, and the QoS requirement can be satisfied if the probability that a sensor locates inside the $r_{0}$-radius disk at the center of each cell is no smaller than $P_{s}$. Observe that the total number $M$ of cells is given by

$$
M=\left\lceil\frac{2}{3 \sqrt{3}\left(r-r_{0}\right)^{2}}\right\rceil,
$$

we choose $r_{0}$ as

$$
r_{0} \triangleq \min \left\{r_{0}: r_{0}<r,\left(1-e^{-\lambda \pi r_{0}^{2}}\right)^{M} \geq P_{s}\right\}
$$

which follows directly from the fact that the numbers of sensors inside disjoint areas are independent for Poisson distribution. We assume that the sensor network is sufficiently dense ( $\lambda$ sufficiently large) so that the above defined $r_{0}$ exists.

\subsection{Collecting One Packet from Each Cell}

The problem we have now is to design a transmission control scheme for collecting one packet from the center area of each cell, 
where we define center area as the $r_{0}$-radius disk at the center of each cell. To have a low latency, low energy cost MAC protocol, we need to eliminate redundant transmissions and fully exploit the MPR capability of the physical layer.

\subsubsection{Elimination of Redundant Transmissions}

Since only one packet needs to be collected from a cell, only one sensor should transmit. To avoid transmissions from sensors located outside the center area, the mobile agent broadcasts $r_{0}$ and the location of the center of this cell at the beginning of one particular slot. All sensors then calculate their distances to the center of this cell. If a sensor finds out that its distance to the center of the cell is larger than $r_{0}$, it refrains from transmitting. For sensors located inside the center area of this cell, carrier sensing is implemented. Specifically, after a sensor with an energy level $\mathcal{E}$ finds out that it is located inside the center area, it chooses a delay $\tau$ according to a predetermined function $f(\mathcal{E})$ and listens to the channel. It will transmit with its chosen delay if and only if no one transmits before it. Considering the relatively small size of the center area, we assume that the signal propagation delay within the center area of a cell is negligible so that carrier sensing ensures one and only one sensor from the center area transmits. Furthermore, if $f(\mathcal{E})$ is chosen to be a strictly decreasing function of the sensor energy level $\mathcal{E}$, this energy-aware carrier sensing not only eliminates redundant transmissions, but also ensures that the sensor with the highest energy supply in the center area transmits $^{1}$.

\subsubsection{Exploitation of MPR Capability}

After partitioning $\mathcal{D}$ into $M$ cells, the mobile agent queues up all $M$ cells and enables in each slot $N$ cells from the head of the queue. Specifically, at the beginning of a slot, the mobile agent broadcasts the center locations of the first $N$ cells in the queue. At the end of this slot, the mobile agent detects whether this slot is empty or not. An empty slot implies that no sensor is located in the center areas of these $N$ cells ${ }^{2}$. These $N$ cells are thus removed from the queue. On the other hand, if this slot is not empty and $k(k \geq 0)$ packets are successfully received, those $k$ cells from which a packet is received are labeled processed and removed from the waiting queue. This procedure continues until the queue becomes empty (all $M$ cells are processed).

With this structure, the only parameter to be designed is the optimal number $N^{*}$ of simultaneously enabled cells. We choose $N^{*}$ by minimizing the weighted sum of the expected number $E[U]$ of total transmissions and the expected number $E[L]$ of slots (latency) for processing all $M$ cells, i.e.,

$$
N^{*}=\arg \min _{N=1, \cdots, N_{\max }} \alpha E[L \mid N]+(1-\alpha) E[U \mid N], 0 \leq \alpha \leq 1,
$$

\footnotetext{
${ }^{1}$ Assuming zero propagation delay within the center area of a cell, $f(\mathcal{E})$ can be any decreasing function with range $\left[0, \tau_{\max }\right]$, where $\tau_{\max }$ can be any positive number. For significant propagation delay, $f(\mathcal{E})$ needs to be chosen judiciously to ensure the efficiency of this energy-aware carrier sensing. This issue will be addressed in our future work.

${ }^{2}$ The probability of having one or more empty center areas is bounded below $1-P_{s}$. See (9).
}

where $E[U \mid N](E[L \mid N])$ denotes the expected number of total transmissions (slots) when $N$ cells are enabled in each slot, $N_{\text {max }}$ is the maximum number of simultaneously enabled cells to be considered. For typical applications, $N_{\max }$ can be set to $n_{0}$ defined in (3). .

In order to determine $N^{*}$, we calculate $E[L \mid N]$ and $E[U \mid N]$ based on a finite state discrete Markov chain. It can be shown that the number of unprocessed cells at the beginning of a slot along with the number of packets that will be transmitted in this slot forms a Markov chain. Specifically, at the beginning of a slot, the network is in state $(j, k)$ if there are $j(j=0, \cdots, M)$ unprocessed cells and $k(k=0, \cdots, \min \{N, j\})$ packets to be transmitted in this slot when the size of access set is chosen to be $N$. A state diagram of this Markov chain for $M=2$ and $N=1$ is illustrated in Figure 2, where $q=1-e^{-\lambda \pi r_{0}^{2}}$ is the probability that a cell has an nonempty center area. With probability $q$, the first cell in the queue has a packet to transmit. Thus, with probability $q$, the Markov chain starts with state $(2,1)$, and with probability $1-q$, it starts with state $(2,0)$. Take state $(2,1)$ for example. With probability $C_{1,0}$, the transmission by the first cell in the queue does not succeed. The chain then stays in $(2,1)$. With probability $C_{1,1} q$, the transmission by the first cell succeeds and the second user in the queue has a packet. The chain then jumps to state $(1,1)$. With probability $C_{1,1}(1-q)$, the chain jumps to state $(1,0)$.

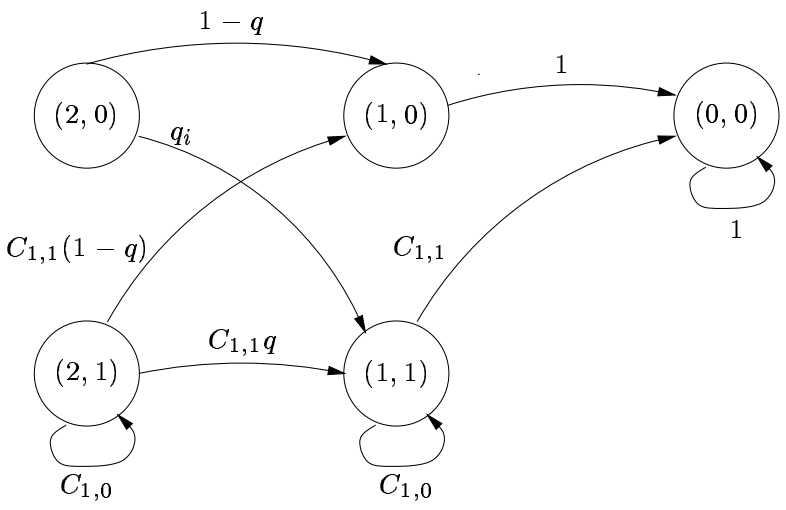

Fig. 2: A state transition diagram.

In general, the transition probability from state $(j, k)$ to state $(l, m)$ is given by

$r_{(j, k),(l, m)}=\left\{\begin{array}{l}B(m, \min \{N, l\}, q) \\ (\text { if } k=0, l=\max \{j-N, 0\}, 0 \leq m \leq \min \{N, l\}) \\ C_{k, j-l} B(m-k+j-l, \min \{j-l, \max \{j-N, 0\}\}, q) \\ (\text { if } 1 \leq k \leq \min \{N, j\}, j-k \leq l \leq j, \\ k-(j-l) \leq m \leq \min \{k, l\}) \\ 0 \quad \text { (otherwise) }\end{array}\right.$

where $B(u, U, s)$ denote the probability mass at the value $u$ of a Binomial random variable with total $U$ trials and a success probability $s$. The initial condition of this Markov chain is given by

$$
P\left[X_{0}=(M, k)\right]=B(k, N, q), k=0, \cdots, N,
$$

where $X_{0}$ denote the initial state of the Markov chain. With state $(0,0)$ defined as the absorbing state, $E[L \mid N]$ is the absorbing 
time of this Markov chain, which is defined as the expected number of transitions until the first hit of state $(0,0)$. Define $e_{(j, k)}$ and $u_{(j, k)}$ as, respectively, the expected remaining time and the expected number of remaining transmissions until absorption given that the current state is $(j, k)$. Let

$$
\begin{aligned}
\mathbf{e} \triangleq\left[e_{(M, 0)}, \cdots, e_{(M, N)}, e_{(M-1,0)}, \cdots, e_{(1,0)}, e_{(1,1)}\right]^{t} \\
\mathbf{v} \triangleq\left[u_{(M, 0)}, \cdots, u_{(M, N)}, u_{(M-1,0)}, \cdots, u_{(1,0)}, u_{(1,1)}\right]^{t} .
\end{aligned}
$$

We then have (see [14] for analysis on the absorbing time of Markov chains)

$$
\mathbf{e}=(\mathbf{I}-\mathbf{P})^{-1} \mathbf{1}, \mathbf{u}=(\mathbf{I}-\mathbf{P})^{-1} \mathbf{k},
$$

where $\mathbf{P}$ is the transition probability matrix (after removing state $(0,0)$ ) with entries specified by $(11), \mathbf{I}$ and $\mathbf{1}$ denote, respectively, an identity matrix and a vector with all entries equal to $1, \mathbf{k} \triangleq[0, \cdots, N, 0, \cdots, N, \cdots, 0,1]^{t}$ is the vector consists of the second indexes of all states. Considering the initial condition of the Markov chain given by (12), we have

$$
\begin{aligned}
& E[L \mid N]=\sum_{k=0}^{N} B(k, N, q) e_{(M, k)}, \\
& E[U \mid N]=\sum_{k=0}^{N} B(k, N, q) u_{(M, k)} .
\end{aligned}
$$

With $E[L \mid N]$ and $E[U \mid N]$ computed for all possible $N$, the optimal number $N^{*}$ of the simultaneously enabled cells can be easily obtained from (10).

We point out that $N^{*}$ can be computed off line; little on-line computation is required to implement the proposed protocol.

\section{SIMULATION EXAMPLES}

In this section, we present numerical examples to demonstrate the performance of QUIRE. Consider a multi-user physical layer with MPR capability provided by spread spectrum. Specifically, each transmitted packet is spread by a randomly generated code with length $P=32$. At the mobile agent, the spreading code of each transmitted packet is assumed known, and a bank of matched filters are used as the receiver. We assume that each packet contains $L_{p}=200$ bits. A block error control code is used which corrects up to $t=2$ errors in each received packet. We consider a noisy environment where the variance of the additive white Gaussian noise denoted by $\sigma^{2}$ is given by $10 \log _{10} \frac{1}{\sigma^{2}}=10 \mathrm{~dB}$. The reception matrix $\mathbf{C}$ for such a network can be easily constructed under the Gaussian assumption on the multiaccess interference from users with equal power (details see [15]). The capacity of the MPR channel in such a network is 6.2327 , which can be achieved by transmitting $n_{0}=8$ packets in each slot.

The random field is a $200 \mathrm{~m} \times 200 \mathrm{~m}$ square. The correlation function $R(d)$ of the random field and the maximum distortion $D$ are such that $r$ as defined in (8) is $10 \mathrm{~m}$. The required probability of reconstructing the random field with a maximum distortion $D$ is given by $P s=0.9$. With $\lambda=1$, the resulting total number of cells is $M=217$.
In Figure 3 we compare the performance of QUIRE with that of a modified TDMA and slotted ALOHA with optimal transmission probability. The modified TDMA is built upon the cell structure and the energy-aware carrier sensing scheme of QUIRE, i.e., cells are enabled one by one in each slot and only the sensor with the highest energy level in the center area transmits. For slotted ALOHA, it is implemented as follows. At the beginning of data collection, the mobile agent, based on the total number of sensors in the field, chooses the optimal transmission probability $P_{t}$ by maximizing the expected number of successful receptions. It than broadcast $P_{t}$ and all sensors flip a coin with bias $P_{t}$ to determine whether to transmit in this slot. At the end of this slot, the mobile agent broadcast the location of all sensors whose packets are successfully received in this slot. All sensors within $r$ distance of these successful sensors will go to sleep; they will not transmit in the future slots of this data collection. At the beginning of the second slot, the mobile agent, assuming the total number of active sensors (excluding all those within $r$ distance of successful sensors), chooses and broadcasts the optimal transmission probability $P_{t}$ for this slot. This procedure continues until the whole network is covered (the number of active sensors becomes 0 ). Note that we intentionally favor slotted ALOHA by assuming the knowledge of active sensors in each slot, while QUIRE only assumes the network density $\lambda$. The performance measure we use here is the weighted sum of expected number of transmissions and expected number of slots for processing all $M$ cells. As shown in Figure 3, QUIRE provides significant performance improvement over the variant versions of TDMA and slotted ALOHA.

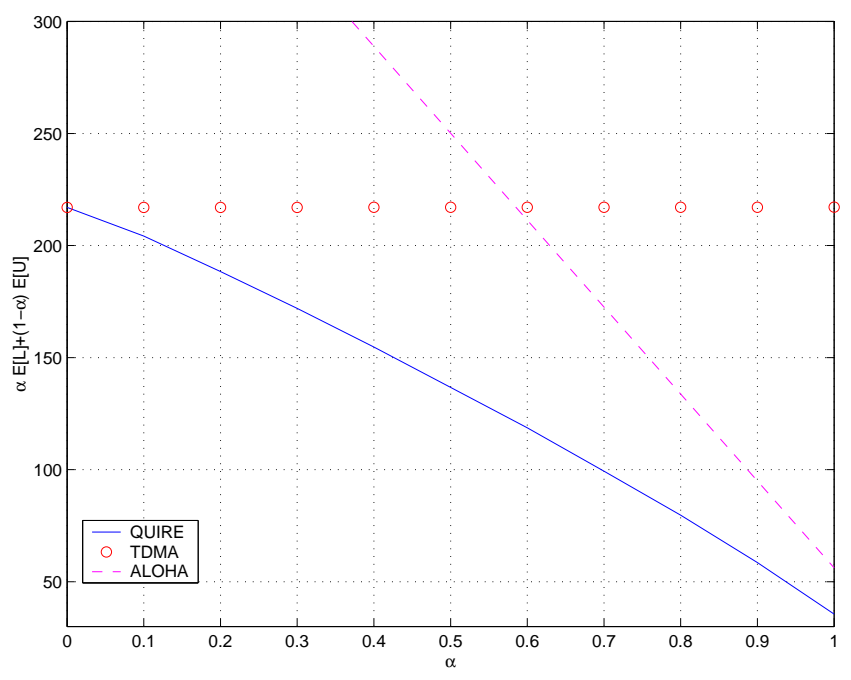

Fig. 3: Performance comparison.

\section{CONCLUSION}

In this paper, we propose QUIRE, a MAC protocol for large scale, densely deployed sensor networks. QUIRE assures quality-ofservice (QoS) requirement with a minimum amount of transmissions from sensors. It completely eliminates redundant transmissions by partitioning the sensor field into disjoint and equal- 
sized cells and enabling only one sensor in each cell to transmit. Built upon a multi-user physical layer which allows simultaneous packet receptions at the mobile agent, QUIRE jointly minimizes system latency and total energy consumption.

\section{REFERENCES}

[1] L. Tong, Q. Zhao, and S. Adireddy, "Sensor Networks with Mobile Agents.” Proc. 2003 MILCOM, Oct. 2003.

[2] A. Woo and D. Culler, "A transmission control scheme for medium access in sensor networks," in Proc. ACM/IEEE Conference on Mobile Communication and Networking, (Rome, Italy), pp. 221-235, July 2001.

[3] W. Ye, J. Heidemann, and D. Estrin, "An Energy-Efficient MAC Protocols for Wireless Sensor Networks," in Proc. of IEEE INFOCOM, (New York, NY), pp. 1567-1576, June 2002.

[4] K. Sohrabi, J. Gao, V. Ailawadhi, and G. Pottie, "Protocols for selforganization of a wireless sensor network," IEEE Personal Communication, pp. 16-27, October 2000.

[5] J. Akyildiz, W. Su, Y. Sankarasubramaniam, and E. Cayirci, "A Survey on Sensor Networks," IEEE Communications Magzine, vol. 40, pp. 102-114, August 2002.

[6] W. Heinzelman, A. Chandrakasan, and H. Balakrishnan, "EnergyEfficient Communication Protocols for Wireless Microsensor Networks," in Proceedings of Hawaaian Intl Conference on Systems Science, (Hawaai,US), January 2000.

[7] Z. Cheng, M. Perillo, B. Tavli, W. Heinzelman, S. Tilak, and N. Abu-Ghazaleh, "Protocols for Local Data Delivery in Wireless Microsensor Networks," in Proc. of the 45th IEEE Midwest Symposium on Circuits and Systems, August 2002.

[8] S. Lindsey, C. Raghavendra, and K. Sivalingam, "Data Gathering in Sensor Networks Using the Energy*Delay Metric," in Proc. of International Workshop on Parallel and Distributed Computing Issues in Wireless Networks and Mobil Computing, (CA), April 2001.

[9] R. Iyer and L. Kleinrock, "QoS Control for Sensor Networks.” To appear in ICC 2003, May 2003.

[10] S. Adireddy, P. Venkitasubramaniam, and L. Tong, "Opportunistic ALOHA and Cross Layer Design for Sensor Networks," in Proc. 2003 MILCOM, (Boston, MA), Oct. 2003.

[11] N. Bulusu, J. Heidemann, and D. Estrin, "Adaptive Beacon Placement," in Proc. of the 21 st International Conference on Distributed Computing Systems, (Arizona), pp. 489-498, April 2001.

[12] L. Doherty, K. Pister, and L. Ghaoui, "Convex Position Estimation in Wireless Sensor Networks," in Proc. of 2001 IEEE INFOCOM, 2001.

[13] A. Savvides, C. Han, and M. Strivastava, "Dynamic Fine-Grained Localization in Ad-Hoc Networks of Sensors," in Proc. of the 7th Annual International Conference on Mobile Computing and Networking, (Rome, Italy), pp. 16-21, July 2001.

[14] D. Isaacson, Markov Chains, Theory and Applications. New York: Wiley, 1976.

[15] Q. Zhao and L. Tong, "A dynamic queue protocol for multiaccess wireless networks with multipacket reception." submitted to IEEE Transactions on Wireless Communications, Nov., 2002, http://people.ece.cornell.edu/ltong/. 